\title{
AUTOMOBILE DRIVING WITH SEVERE AMNESIA
}

\author{
Nicole Skaar \\ Steven W. Anderson \\ Jeffrey Dawson \\ Matthew Rizzo \\ Colleges of Medicine and Public Health \\ Public Health Center \\ University of Iowa \\ Iowa City, Iowa, USA \\ E-mail: matthew-rizzo@uiowa.edu
}

\begin{abstract}
\section{OBJECTIVE}

Examine effects of severe memory impairment on driving an automobile.

\section{BACKGROUND}

Automobile driving is a complex task requiring coordination of many cognitive and visuomotor processes. Disordered memory is a common result of many diseases including stroke, traumatic brain injury, and herpes simplex encephalitis. Amnesics may forget the rules of the road, the location of controls or surrounding vehicles, and forget to fasten seat belts, check mirrors and gauges, and to fuel or service the car. These drivers may fail to recognize key landmarks, misinterpret previously familiar road signs or symbols, and are at high risk of getting lost.
\end{abstract}

\section{DESIGN/METHODS}

We studied two men (ages 46 and50) who continued to drive despite severe persistent memory impairments and bilateral mesial temporal lesions due to herpes encephalitis. Standardized neuropsychological tests were used to evaluate cognitive abilities. We used ARGOS, a state-ofthe-art instrumented vehicle with extensive instrumentation and sensors hidden within its infrastructure, to measure driver performance on tasks that challenged critical cognitive abilities during driving. Measures of speed and steering were recorded at a rate of $10 \mathrm{~Hz}$. The road test in ARGOS lasted approximately 45 minutes.

\section{RESULTS}

Serial neuropsychological testing confirmed severe and stable memory impairments (e.g., AVLT and CFT- 30 recall) with preserved abilities in other cognitive domains. Snellen visual acuity and Pelli-Robson contrast sensitivity were normal. Neither amnesic could learn the directions for a navigation task in ARGOS, yet both could complete the necessary turns with assistance from an on-board experimenter. Both also showed difficulty on a landmark identification task. Yet, both amnesics showed good speed and steering control compared to 59 neurologically normal controls of similar age (mean age 49.8), and committed no hazardous errors. 


\section{CONCLUSIONS}

Despite profound amnesia, both subjects could learn and retain the procedural knowledge necessary to handle an unfamiliar vehicle, and both remembered road rules and locations of surrounding vehicles. Yet neither could learn and follow directions for the navigation task in ARGOS, consistent with inability to learn and recall new declarative information. Their good speed and steering underscores that the automatic processes of attention that allow a driver to control a vehicle can remain intact despite profound memory impairment. Also, measures of driver visuomotor control (steering and lane position variability) alone are not sensitive predictors of critical incidents caused by cognitively impaired drivers. Despite being able to handle a vehicle, both amnesics had trouble remembering the destination and purpose of the trip. There would be no reason to revoke the license of someone with isolated severe memory impairment from herpes encephalitis; however, license restriction (e.g., driving only while accompanied) may be advisable to mitigate problems caused by getting lost.

Study supported by NIA AG 15071 and NIA AG 17717. 